\title{
Guidelines for the characterization and use of fibre optic sensors - Basic definitions \& a proposed standard for FBG-based strain sensors
}

\author{
Wolfgang R. Habel*1,a,b ${ }^{\text {Ingolf Baumann }}{ }^{2, b}$, Francis Berghmans ${ }^{3, a}$, Krzysztof Borzycki ${ }^{4, a}$, Christoph \\ Chojetzki $^{5, \mathrm{~b}}$, Karl-Heinz Haase ${ }^{6, \mathrm{a}, \mathrm{b}}$, Leszek R. Jaroszewicz ${ }^{7, \mathrm{a}}$, Thomas Kleckers ${ }^{6, \mathrm{~b}}$, Marc Niklès ${ }^{8, \mathrm{a}}$, \\ Manfred Rothhardt ${ }^{9, b}$, Vivien Schlüter ${ }^{1, b}$, Luc Thévenaz ${ }^{10, a}$, Moshe Tur ${ }^{11, a}$, Marc Wuilpart ${ }^{12, a}$ \\ ${ }^{1}$ BAM Federal Institute for Materials Research and Testing, Unter der Eichen 87, D - 12205 Berlin/Germany; \\ ${ }^{2} \mathrm{AOS}$ GmbH Dresden/Germany; ${ }^{3}$ Vrije Universiteit Brussel/Belgium; ${ }^{4}$ National Institute of Telecommunica- \\ tions/Poland; ${ }^{5}$ FBGS Technologies GmbH Jena/ Germany; ${ }^{6} \mathrm{HBM}$ GmbH/ Germany; ${ }^{7}$ Military University of \\ Technology/Poland; ${ }^{8}$ Omnisens SA/Switzerland; ${ }^{9}$ IPHT Jena/ Germany; ${ }^{10}$ Ecole Polytechnique Fédérale de \\ Lausanne/Switzerland; ${ }^{11}$ Tel-Aviv University/Israel; ${ }^{12}$ Faculté Polytechnique de Mons/Belgium
}

\begin{abstract}
This paper describes the outcome of two groups which are involved in the specification of guidelines for fibre optic sensors performance and testing. The "Guideline for use of fibre optic sensors" from the COST-299 guideline group, and the "Optical Strain Sensor based on Fibre Bragg Grating" from the GESA guideline group of the VDI - "The Association of German Engineers". Through appropriate specifications and definitions, both guidelines aim at enabling better understanding of fibre optic sensors characteristics and performances. A concise view into the structure of the guidelines is presented, emphasizing important aspects. The English version of the two guidelines will be available in autumn 2009.
\end{abstract}

Keywords: Guideline, standards, fibre optic sensor, strain sensor, fibre Bragg grating, distributed sensor

\section{INTRODUCTION}

Development of standards and guidelines for performance specifications and testing for fibre optic sensors has been discussed since the mid-nineties of the last century in the scientific community as well as in the industry. Very global standards for the use of fibre optic components in data communication and telecommunication have been available for more than 20 years. Guidelines or substantial standards for fibre optic sensors are rather an exception. The first standard draft on generic specification of fibre optic sensors has been published in 1995 [1]; the first draft for a specific type of fibre sensor - the fibre optic gyroscope - was published in 1996 [2]. Some terms used in fibre optic communication are quite close to the terminology typically used in fibre optic sensor technology. However, there is a huge number of specific issues associated with specifically fibre optic sensing systems. These items are not considered in existing guidelines or standards. For instance, standards for fibre optic sensors have to cover characteristic details related to the respective physical sensor mechanism, to the sensor response for different measurands, to the application, and finally to specific environmental conditions.

Naturally, it is not possible to cover either all different aspects of fibre optic sensors in one standard or a set of harmonized standards. This very complex matter requires specific guidelines for specific sensor types (e.g. distributed sensors, point sensors such as fibre Bragg grating (FBG) sensors, sensors for mechanical measurands such as strain, deformation, biological and chemical sensors or sensors for physical quantities such as pressure, humidity, and ionizing radiation).

In order to define clear guidelines and/or regulations for appropriate characterization performance specifications and better understanding of frequently used fibre optic sensors, two particular activities have been established in Europe over the past four years. One activity has been established within the European COST Action 299 "FIDES" (Optical Fibres for New Challenges Facing the Information Society) in the framework of its Working Group 4: "New Challenges in Fiber Optic Sensors". The other activity has been established in the German "Association for Experimental Stress Analysis" (GESA) within the VDI - "The Association of German Engineers". The particular contents of the guideline papers worked out in these two groups are presented (see [3] for another initiative in this area).

*wolfgang.habel@bam.de; phone +49 30 8104-1916; fax +49 30 8104-1917; www.bam.de

${ }^{\mathrm{a}}$ Member of COST 299 guideline group, ${ }^{\mathrm{b}}$ Member of German guideline group within VDI - The Association of German Engineers

20th International Conference on Optical Fibre Sensors, edited by Julian Jones, Brian Culshaw, Wolfgang Ecke, José Miguel López-Higuera, Reinhardt Willsch, Proc. of SPIE Vol. 7503, 75035E (C) 2009 SPIE · CCC code: 0277-786X/09/\$18 - doi: 10.1117/12.835362 


\section{COST-299 GUIDELINES FOR THE USE OF FIBRE OPTIC SENSORS}

\subsection{Background}

The COST Action 299 "FIDES" (Optical Fibres for New Challenges Facing the Information Society), established in 2005 consolidates a respectable number of fibre sensor experts from all over Europe, from very different topics and application fields. This platform provided an excellent basis for the discussion of terms and very important details for appropriate use of fibre optic sensors.

During the formation of the COST Action 299, the Section Group 3 (SG3) of the Working Group (WG4) "New Challenges in Fiber Optic Sensors" [4] has taken upon itself to develop guidelines and standards for preferably long gauge length sensors. Application issues specific for fibre optic sensors were identified as an important part of the newly founded COST action. Because the appropriate design and use of fibre optic sensor systems require undoubtedly clear definitions of sensor-specific terms, clear information on sensor characteristics, including limitations for use, clear definition of relevant manufacturing and testing procedures, and last but not least proof test conditions, WG4/SG3 has assembled an expert Group to formulate basic terms and describe basic sensor categories. Because of the limited time within the COST action and due to the very complex matter, first, a consensus on the definition of basic terms and on the description of important sensor features was targeted.

\subsection{Structure of the guidelines}

The COST 299 guidelines draft is being circulated among various expert groups (in parallel with this paper submission) with the objective to get critical comments. The structure of the guidelines, including the following sub items is:

1. Introduction

2. General terms; this category describes terms affecting all or most fibre optic sensors: type of used fibre, type of sensor, distance range, measurement range, wavelength of operation, characteristic wavelength @ reference temperature (FBGs), gauge factor / scale factor @ reference conditions, true value.

3. Functionality; this category describes terms useful while working with fibre optic sensors: fatigue, life expectancy/lifetime, durability, failure criteria, gauge length, sampling interval, optical power dynamic range (optical budget), warm-up time, measuring time, updating time, limiting conditions.

4. Response characteristics; this category describes terms which give correlation between output quantities of a measurement system and the corresponding quantitative characteristics of the measurand: resolution, spatial resolution, measurement dynamic range, scale factor, responsivity, linearity, drift, cross-sensitivity, full scale.

5. Quantities of random nature; this category describes terms specifying unpredictable variations in measurement results affecting system reliability: accuracy, location accuracy, distance accuracy, precision, repeatability, reproducibility, uncertainty of measurement, bias, noise, stability.

6. Optical safety related quantities; the cited guidelines refer to safe use of fibre optic sensors with laser-based interrogators.

7. Sources, references.

\subsection{A selected example: Resolution}

The spirit and purpose of these guidelines is to be illustrated by an example ("resolution"). Not only users of fibre optic distributed sensors are confused by important terms like resolution, even experts have different views what this term actually means and how to precisely define and measure it. The terms defined in the guideline are the outcome of extensive and engaged discussions to provide generally acknowledged understanding of important fibre optic sensor terms. When adopted, the proposed definitions will facilitate a more objective and precise comparison of the characteristics of different devices.

Fig. 1. Clarification of the term "resolution"

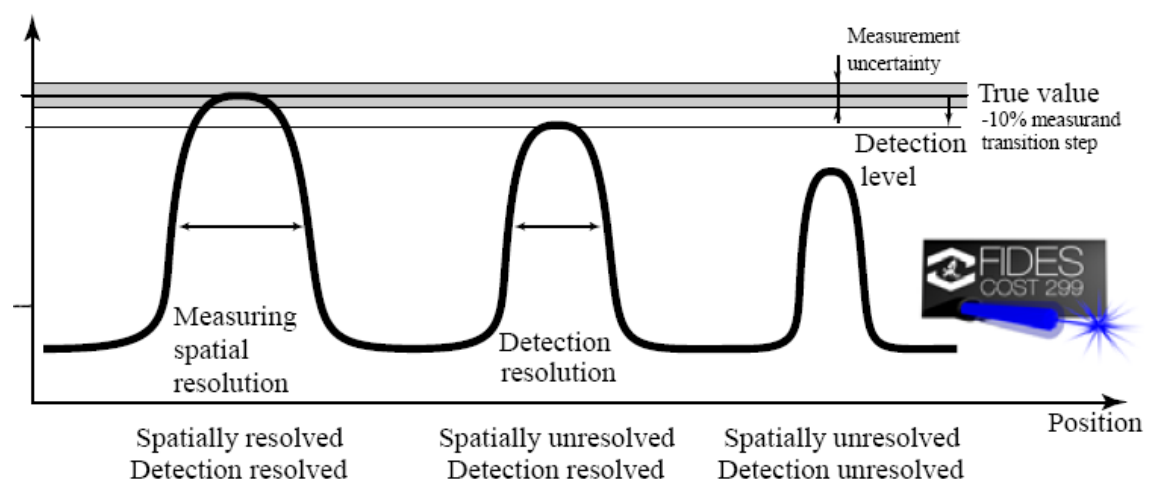


According to the given definition, Resolution represents the smallest change in the measurand, meaningfully detectable by the measurement system. The resolution is limited by either the instrument readout or precision $(\sigma)$, whichever is bigger. Next, the following derivatives definitions are presented: Spatial resolution is specified for a fiber by the minimum distance between two step transitions of the measurand of at least 20 times its resolution. Measuring spatial resolution results in the minimum distance over which the system is able to indicate the value of the measurand within the specified uncertainty. Detection spatial resolution results in the minimum distance that generates readings that are within $10 \%$ of the measurand transition amplitude.

\section{VDI/VDE GUIDELINE 2660: OPTICAL STRAIN SENSORS BASED ON FIBRE BRAGG GRATING}

\subsection{Background}

One of the expert committees in the German Association for Experimental Stress Analysis (GESA) within the VDI - The Association of German Engineers is the Working Group AK17: "Fiber Optic Measurement Methods" [5]. Among its other activities, this committee accommodates a platform to discuss application-oriented aspects and to present new results, related to the use of fibre sensors. The development of a standard for reliable manufacturing, testing and use of fibre Bragg grating strain sensors is of particular significance for the core members of this expert committee. Because of the large number of companies which offer FBG sensors for a wide range of applications, standardization of the testing methods and standards for the communication of the sensor characteristics was urgently needed and has been developed in the past year. This guideline covers the most important terms and aspects for manufacturers and users alike. The standard has the English title "Experimental Stress Analysis - Optical Strain Sensor based on fibre Bragg grating; Basics, Characteristics and its Testing" and is initially a German VDI/VDE guideline, which was published in April 2009. VDI/VDE guidelines are generally regarded as highly regarded recommendations. Such guidelines find an interested community and are considered as generally recognized engineering standards. The VDI core group for development of the FBG strain sensor guidelines consists of experts from three German companies and two research institutes; the members are marked by the superscript $b$ in the authors list.

\subsection{Structure of the guideline}

The VDI/VDE 2660 guideline was drawn up with the objective to provide directive technical and scientific procedural documentation and decision-making aids for manufacturers, distributors and users of FBG strain sensors. It treats significant technical questions from scientific and economic standpoints and formulates assessment and evaluation criteria that facilitate the technology transfer of new products. Important addressed issues are:

- Terms and definitions

- Design-specific features and characteristics to be measured

- Recurring specifications for characteristics testing

- Test devices requirements for use in test facilities

- Communication of the measurement results for investigated characteristics.

Basically, optical strain sensors (OSS) based on FBG sensors can be used in three different engineering designs: a) as a fibre with one or several sensors along its length (FBG array), b) as extensometer sensor whereas the fibre ends of the grating are fixed at two points with definite distance, and c) as a FBG patch, where the grating is embedded in a protective layer or directly fixed to a carrying frame. Commonly, the planar patch is fixed to the measured object. These three design versions are considered and basic terms are defined, such as gauge length, strain, reference strain, wavelength reference, stability criteria for the optical strain sensor, zero point- related and non-zero point-related measurement.

\subsection{Aspects of special significance}

An important point of the guideline concerns design-specific features which affect the characteristics of the sensor. Significant parameters and terms are therefore considered and defined: e. g. Bragg wavelength, spectral width, reflectivity, strain sensitivity (k-factor characteristics), ultimate strain for room temperature, fatigue behaviour, minimum bending radius, response to temperature changes. It is well-known that the reproducibility of measurements using FBG sensor systems not only depends on the quality of the Bragg grating spectra but also on the stability and reproducibility of the wavelength measurement with the employed recording devices. One aspect concerns parameters of the FBG that define the Bragg wavelength. It is necessary to know the set-up wavelength of the grating. The determination of the 
Bragg wavelength using a Full Width at Half Maximum (FWHM) algorithm can differ from a Bragg wavelength determined by a centroid calculation algorithm. This is due to the influence of the grating characteristics (mainly uniformity of the spectral shape, amplitude) and the sampling density of the FBG spectrum, as well as on the uncertainty of the curve fit algorithm for the determination of the Bragg wavelength [6], [7]. The guideline recommends which parameters and terms have to be specified in order to record reliable measurement results.

Another important section of the guideline focuses on the requirements for certified testing facilities used to characterize FBG-based sensors. In order to ensure reliable measurements with such sensors, requirements on their recording devices are also defined, such as requirements on the estimation of the Bragg wavelength, spectral width, reflectivity, strain sensitivity and the other previously defined terms. Recommendations are also given on how to communicate the measured results. The guideline refers to the GUM [8], as well as to the standard [9], which ensures the use of calibrated and validated testing facilities. Finally, the guideline presents a model of a data sheet, which lists a number of functional characteristics that have to be specified, such as Bragg wavelength, k-factor characteristics, response to temperature changes, spectral width, and reflectivity. The model data sheet also recommends a number of other important specific parameters and design specifications that provide comprehensive information for designers and users of such sensor systems. In any case, manufacturers and users who observe the recommendations of this guideline are allowed to sell their products with the cachet: "Qualified according to guideline VDI/VDE 2660".

\section{SUMMARY}

In the European fibre optic sensor community, important work aiming at the creation of guidelines has been carried out. Basic terms have been defined in such a way so that all characteristic parameters of optical strain sensors based on fibre Bragg gratings, can be uniquely measured and certified. The guidelines definition work will continue to develop recommendations on how to ensure appropriate application procedures, considering the micromechanical influence of the materials in the sensing area (material of the measuring object, adhesives, protecting layers, coatings), as well as chemical and physical interactions of all of these materials under changing environmental conditions. Calibration and validation facilities will be recommended to evaluate all the effects which influence the measurement results, and which help to get reliable sensor signals over a long period of operation.

The drafts of these guidelines will be modified after gathering comments and feedback during the summer of 2009 and then published as a COST 299 report, accessible via the COST website [4], and, respectively, as VDI/VDE guideline 2660. Both final versions will be available (in English) by October 2009.

\section{ACKNOWLEDGMENT}

The authors would like to pay tribute and to express their warm thanks for the continuous, outstanding, friendly and very effective cooperation in both groups. Sincere thanks are given to COST office in Brussels, VDI in Germany as well as to all institutions and companies that supported the national and European guideline work.

\section{REFERENCES}

[1] IEC 61757-1:1995. Fibre Optic Sensors - Part 1: Generic Specification.

[2] IEEE Standard Specification Format Guide and Test Procedures for Single-Axis Interferometric Fiber Optic Gyros. Working Draft P952/D24 (December 1996).

[3] Habel, W.R.: Reliable Use of Fiber Optic Sensors. In: Encyclopedia of Structural Health Monitoring. (Eds: Boller, C. et al.). John Wiley \& Sons. 1. Edition January 2009. ISBN-10: 0-470-05822-6, ISBN-13: 978-0-470-05822-0

[4] COST 299 information: http://www.cost299.org, 2009

[5] The Association of German Engineers (VDI), Society of Experimental Stress Analysis (GESA). http://www.vdi.de/strukturmonitoring, 2009.

[6] Dyer, S. D.; Key metrology considerations for fiber Bragg grating sensors. SPIE vol. 5384 (2004), 181-189.

[7] A. F. Fernandez, A. Gusarov, F. Berghmans, K. Kalli, V. Polo, H. Limberger, M. Beukema and P. Nellen: Roundrobin for fiber Bragg grating metrology during COST270 action. SPIE vol. 5465 (2004), 210-216.

[8] Guide to the Expression of Uncertainty in Measurement (GUM). ISO, First Edition 1995.

[9] Standard EN ISO/IEC 17025:2000 (trilingual version): General requirements for the competence of testing and calibration laboratories (ISO/IEC 17025:1999). 